\title{
Design of Unconstrained DMC to Improve the Distillate Product Purity of the Distillation Column
}

\author{
S. Bhat Vinayambika ${ }^{1}$, I. Thirunavukkarasu ${ }^{1}$, and S. Shanmuga Priya ${ }^{2}$ \\ ${ }^{1}$ Dept. of Instrumentation and Control Engineering, Manipal Institute of Technology, Manipal University, Karnataka-India \\ ${ }^{2}$ Dept. of Chemical Engineering, Manipal Institute of Technology, Manipal University, Karnataka-India
}

\begin{abstract}
This paper demonstrates the use of unconstrained Dynamic Matrix Control (DMC) to control the process transfer function with time delay. The selection of tuning parameter is challenging task in predictive control algorithm. The DMC scheme is designed and it is used here to control the process transfer function, First Order Plant with Dead Time (FOPDT) model. Here, one manipulated variable (reflux) and one controlled variable (distillate) is considered for the implementation. The algorithm significantly controls the reflux to improve the distillate product purity. The simulation is done using MATLAB m-file. Both servo and regulatory responses were obtained. The simulation result validates the effectiveness of the proposed algorithm.
\end{abstract}

\section{Introduction}

The predictive control approach is a popular technique for the control of slow dynamical systems, such as those encountered in chemical process control in the petrochemical, pulp and paper industries, gas pipeline control, waste heat boiler, and active vibration control of railway vehicle [1]. It was developed by Shell Oil Company in 1980's. One of the basic predictive algorithm is Dynamic Matrix Control (DMC) is presented here for Single Input Single Output (SISO) system [2]. DMC has the capability to handle constraints on the manipulated and controlled variables in a systematic manner. The basic elements of DMC are: (i) a model of the plant to predict its future, (ii) a cost function which reflects control objectives, (iii) constraints on inputs and states/outputs, (iv) an optimization algorithm and, (v) the receding horizon principle [2]. The tuning of DMC for FOPDT has been simulated here. The tuning of unconstrained DMC is a challenging task since the number of adjustable parameters that effect closed loop performance. The tuning parameter $(\mathrm{P}, \mathrm{M}$, and $\mathrm{N})$ needs to be selected appropriately to obtain the better performance.

S. Rajasekaran and T. Kannadasan [3] proposed simplified predictive control for controlling a temperature of a fluid stream using the shell and tube heat exchanger. The first order plus time delay transfer function model of heat exchanger have been considered for the study. R. D. Kokate and L. M. Waghmare [4] have presented Internal Model Control (IMC) based DMC control technique to control the heat exchanger process. The aim here is to eliminate the offset brought from load disturbances. Saniye Ay and Sileyman Karacan [5] had proposed the decoupling constrained model predictive control of a multi-component packed distillation column. R. D. Kokate and L. M. Waghmare proposed DMC algorithm for Single Input Single Output (SISO) system. The response of the system depends on the selection of the tuning parameters. There is various tuning technique to identify the parameters $\mathrm{P}, \mathrm{N}$ and $\mathrm{M}$ of the DMC algorithm in literature [6]. A simple First Order Plus Dead Time (FOPDT) model is considered for study and experimentation. S. Srinivasulu Raju et al. [7] designed the dynamic matrix control algorithm for driven pendulum system. The second order transfer function model of the driven pendulum has been considered here to implement the control algorithm. Jose Manuel LopezGuede [8] had studied the effect of the prediction horizon in the performance of a Dynamic Matrix Control (DMC). It is found that low to moderate value of prediction horizon results in a better performance of the DMC controller. Lin Cong et al. [9] developed multivariable DMC for high purity internal thermally coupled distillation column. They have considred feed as benzene/toluene system for the study. Manimaran. M et al. [10] proposed DMC for a pilot scale distillation column.

The paper briefly introduces the Dynamic Matrix Control algorithm. Also the desired system response transfer matrix is proposed and it is simulated using Matlab m-file. The servo and regulatory response is studied here. The DMC can handle the load disturbance rejection effectively. The FOPDT transfer function is considered here for illustration. Results are presented so as to demonstrate the superiority of the proposed method. Finally, conclusions are given in the last section. 


\section{Materials and methods}

\subsection{Conventional dynamic matrix control}

The DMC is the most basic and popular MPC algorithm used in the process control industries. The block diagram representation is as shown in Fig. 1. The DMC adopts step response approach. It works on the principle of receding horizon. The time horizon was moved one step ahead during every control cycle and the optimization problem is solved during every control cycle. The main feature of this predictive control algorithm is its ability to deal with constraints on system variables. Since constraints are usually neglected in many of control algorithms [3].

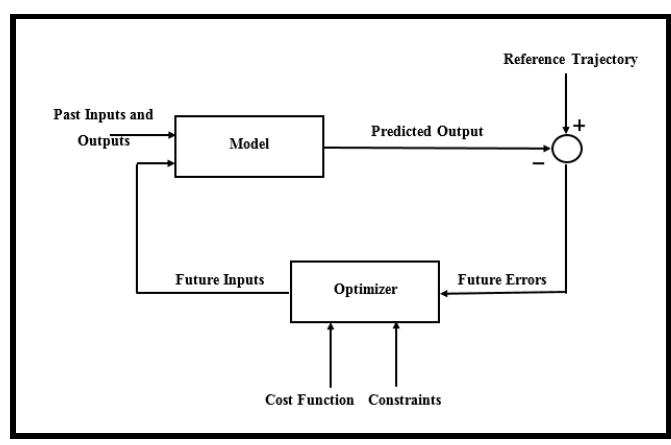

Figure 1. Basic structure of MPC

The process model consists of the information about the controlled process. According to the manipulated variables it predicts its future values. There after minimization of cost function ensures that the error is reduced. In the last step different optimization techniques are applied and the output gives the input sequence for the next prediction horizon.

The DMC algorithm basically has the following steps:

1. Step response model for the given plant.

2. Over finite predictive horizon find the quadratic performance objective.

3. Based on predicted value try to follow the setpoint as closely as possible.

4. Optimal input need to be calculated based on least squares problem.

DMC control is based on a step response model that calculates a desired value of the manipulated value that remains unchanged during the next time step [4]. The new value of the manipulated variable the value that gives the smallest sum of squares error between the setpoint and the predicted value of the controlled variable. The number of time steps the DMC uses for its prediction is called the Prediction Horizon.

A large part of DMC's appeal is drawn from an intuitive use of a finite step response model of the process, a quadratic performance objective over a finite prediction horizon, and optimal manipulated input moves computed as the solution to a least squares problem.

\subsection{Cost function}

The cost function is a measure of performance of the process model. It is also called objective function. It is the performance criterion for controller [6]. The controller will make plant to follow a particular reference trajectory with satisfying performance criterion. This is obtained by minimizing the cost function. All objective function is having subfunctions which are required to be penalized. There are different types of cost functions like standard least square or quadratic objective function and absolute value objective function. The most commonly used objective functions is quadratic objective function. It is a sum of squares of the difference between the setpoints and the model predicted outputs and the control moves. A control move is the change in control action from one step to another step. For a $\mathrm{P}$ step prediction horizon and $M$ step control horizon, the quadratic objective function is given below.

$$
J=\sum_{i=1}^{P}\left(y_{r_{k+i}}-y_{P k+i}\right)^{2}+\lambda\left(\sum_{i=0}^{i=M-1}\left(\Delta u_{k+i}\right)^{2}\right)
$$

where

$\lambda \quad$ : Control weighing function

$\Delta \mathrm{u} \quad$ : Change in the manipulated variable from one sample time to another sample time

$$
\begin{array}{ll}
\mathrm{y}_{\mathrm{r}} & : \text { Reference trajectory } \\
\mathrm{y}_{\mathrm{P}} & \text { : Predicted output } \\
\mathrm{M} & : \text { Model length } \\
\mathrm{P} & \text { : Prediction horizon } \\
\mathrm{k} & \text { : Sampling instants }
\end{array}
$$

\subsection{Derivation of DMC algorithm}

The main motive of Dynamic Matrix Control is to find the input signal that best satisfies to a given performance criterion, it predicts how the system will behave if the signal is applied $[7,11]$. This algorithm uses the samples of step response of plant to capture plant's nature and solves a control problem for an optimal control action to track the given input [8].

Based on the step response model:

$$
y_{k}=\sum_{i=1}^{\infty} s_{i} \Delta u_{k-i}
$$

Since the step response saturates after $\mathrm{N}$ steps:

$$
\text { i.e } s_{N}=s_{N+1}=s_{N+2}=\ldots \ldots \ldots
$$

Thus, substituting (1) into (2) we have,

$$
\begin{gathered}
y_{k}=\sum_{i=1}^{N-1} s_{i} \Delta u_{k-i}+s_{N} \sum_{i=N}^{i=\infty} \Delta u \\
\text { i.e } y_{k}=\sum_{i=1}^{N-1} s_{i} \Delta u_{k-i}+s_{N}\left(u_{k-N}\right)
\end{gathered}
$$

Thus, predicted output at the first time step in the future can be found from:

$$
y_{k+1}=\sum_{i=1}^{N-1} s_{i} \Delta u_{k-i+1}+s_{N}\left(u_{k-N+1}\right)
$$




$$
y_{k+1}=s_{1} \Delta u_{k}+\sum_{i=2}^{N-1} s_{i} \Delta u_{k-i+1}+s_{N} u_{k-N+1}
$$

Hence, for $\mathrm{j}^{\text {th }}$ step in future:

$$
y_{k+j}=\sum_{i=1}^{j} s_{i} \Delta u_{k-i+j}+\sum_{i=j+1}^{N-1} s_{i} \Delta u_{k-i+j}+s_{N} u_{k-N+j}
$$

The above equation can be separated into effects of past and future control moves:

$$
y_{k+j}=s_{1} \Delta u_{k+j-1}+s_{2} \Delta u_{K+j-2}+\ldots \ldots+s_{j} \Delta u_{k}
$$

Effects of current and future moves

$$
\begin{aligned}
& +s_{N} u_{k-N+j}+s_{j+1} \Delta u_{k-1}+s_{j+2} \Delta u_{k-2} \\
& +\ldots \ldots \ldots \ldots \ldots \ldots \ldots+s_{N-1} \Delta u_{k-N+j+1}
\end{aligned}
$$

Effects of past moves

There is no control moves beyond the control horizon M steps, so:

$$
\Delta u_{k+M}=\Delta u_{k+M+1}=\ldots \ldots=\Delta u_{k+P-1}=0
$$

In matrix-vector form, for a prediction horizon $\mathrm{P}$ and a control horizon of $\mathrm{M}$ steps yields:

$$
\begin{aligned}
& {\left[\begin{array}{c}
y_{k+1} \\
y_{k+2} \\
\vdots \\
y_{k+j} \\
\vdots \\
y_{k+P}
\end{array}\right]_{P \times 1}=\left[\begin{array}{cccccc}
s_{1} & 0 & 0 & \cdots & 0 & 0 \\
s_{2} & s_{1} & 0 & \cdots & 0 & 0 \\
\vdots & \vdots & & & & \\
s_{j} & s_{j-1} & s_{j-2} & \cdots & \cdots & s_{j-M+1} \\
\vdots & \vdots & \vdots & & & \vdots \\
s_{P} & s_{P-1} & s_{P-2} & \cdots & \cdots & s_{P-M+1}
\end{array}\right]_{P \times M}\left[\begin{array}{c}
\Delta u_{k} \\
\Delta u_{k+1} \\
\vdots \\
\Delta u_{k+M-2} \\
\Delta u_{k+M-1}
\end{array}\right]_{M \times 1}} \\
& +\left[\begin{array}{cccccc}
s_{2} & s_{3} & s_{4} & \cdots & s_{N-2} & s_{N-1} \\
s_{3} & s_{4} & s_{5} & \cdots & s_{N-1} & 0 \\
\vdots & \vdots & \cdots & & 0 & 0 \\
s_{j+1} & s_{j+2} & \cdots & s_{N-1} & 0 & 0 \\
\vdots & \vdots & \vdots & & & \vdots \\
s_{P+1} & s_{P+2} & \cdots & 0 & \cdots & 0
\end{array}\right]_{P \times(N-2)}\left[\begin{array}{c}
\Delta u_{k-1} \\
\Delta u_{k-2} \\
\vdots \\
\Delta u_{k-N+3} \\
\Delta u_{k-N+2}
\end{array}\right]_{(N-2) \times 1} \quad+s_{N}\left[\begin{array}{c}
u_{k-N+1} \\
u_{k-N+2} \\
\vdots \\
u_{k-N+P}
\end{array}\right]_{P \times 11} \\
& \text { i.e } y_{P}=S_{f} \Delta u+S_{p a s t} \Delta u_{p a s t}+s_{N} u_{P}
\end{aligned}
$$

Thus, the difference between the reference trajectory $\mathrm{y}_{\mathrm{r}}$ and the future prediction $\mathrm{y}_{\mathrm{P}}$ is:

$$
y_{r}-y_{P}=y_{r}-\left\lfloor S_{\text {pas }} \Delta u_{P}+s_{N} u_{P}\right\rfloor-S_{f} \Delta u
$$

The above equation can be written as:

$$
e^{f}=e-S_{f} \Delta u
$$

where the future predicted error is composed of 'free response' (e) and 'forced response' $\left(-\mathrm{S}_{\mathrm{f}} \Delta \mathrm{u}\right)$.

The least square objective function is [9]:

$$
\begin{gathered}
J=\sum_{i=1}^{P}\left(e_{k+i}^{f}\right)^{2}+\lambda \sum_{i=0}^{M-1}\left(\Delta u_{k+i}\right)^{2} \\
\sum_{i=1}^{P}\left(e_{k+i}^{f}\right)^{2}=\left[\begin{array}{lllll}
e_{k+i}^{f} & e_{k+2}^{f} & \ldots & \ldots & e_{k+P}^{f}
\end{array}\right] \times\left[\begin{array}{c}
e_{k+1}^{f} \\
e_{k+2}^{f} \\
\ldots \\
\ldots \\
e_{k+P}^{f}
\end{array}\right]
\end{gathered}
$$

$$
\begin{aligned}
& =\left(e^{f}\right)^{T} e^{f}
\end{aligned}
$$

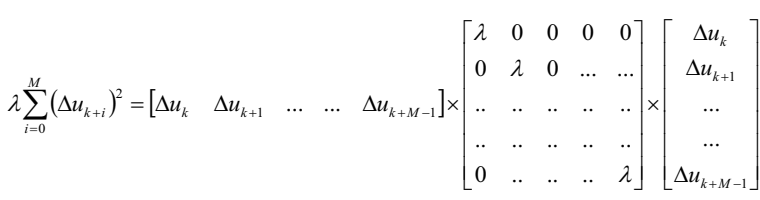

$$
\begin{aligned}
& =\Delta u^{T} \lambda \Delta u
\end{aligned}
$$

Thus, objective function is written in the form:

$$
J=\left(e^{f}\right)^{T} e^{f}+(\Delta u)^{T} \lambda(\Delta u)
$$

The objective function is subjected to the modelling equation equality constraint:

$$
e^{f}=e-S_{f} \Delta u
$$

On expanding the objective function,

$$
\begin{gathered}
J=\left(e-S_{f} \Delta u\right)^{T}\left(e-S_{f} \Delta u\right)+(\Delta u)^{T} \lambda \Delta u \\
J=e^{T} e-S_{f}^{T} \Delta u^{T} e-e^{T} S_{f} \Delta u+\Delta u^{T} S_{f}^{T} S_{f} \Delta u+\Delta u^{T} \lambda \Delta u \\
J=e^{T} e-2 S_{f}^{T} \Delta u^{T} e+\Delta u^{T}\left[S_{f}^{T} S_{f}+\lambda\right] \Delta u
\end{gathered}
$$

Thus, DMC control law solution for the minimization of the above given optimization function is given by solving $\frac{\partial J}{\partial \Delta u}=0$ and hence find $\Delta \mathrm{u}$.

$$
\begin{gathered}
\frac{\partial J}{\partial \Delta u}=0-2 S_{f}^{T} e+2\left(S_{f}^{T} S_{f}+\lambda\right) \Delta u=0 \\
\Delta u=\left(S_{f}^{T} S_{f}+\lambda\right)^{-1} S_{f}^{T} e
\end{gathered}
$$

Thus, $\Delta u=K e$

where $K=\left(S_{f}^{T} S_{f}+\lambda\right)^{-1} S_{f}^{T}$ and $e=y_{r}-y_{P}$

Only one control move is actually implemented to calculate the $\Delta \mathrm{u}$, thus only first row of $\mathrm{K}$ matrix is considered [10].

$$
\text { i.e } \Delta u_{k}=K_{1} E
$$

The flow chart of Dynamic Matrix Control algorithm is given in Fig. 2 as shown below.

\subsection{DMC tuning}

The DMC design technique has some design parameters ( $\mathrm{P}, \mathrm{M}$ and $\mathrm{N}$ ), which can be tuned to get the desired response of the controller. These parameters are called the tuning parameters of the controller. The following guidelines are basically used to tune a DMC.

- The model length $(\mathrm{N})$ should be approximately the open loop time of the process. When using step response model, it is important to make certain that the model 
length $(\mathrm{N})$ is long enough to capture the steady state change [12].

- P determines how far into the future the control objective reaches and thus includes the main dynamic characteristics of the target. If $\mathrm{P}$ is increased results in more gradual control action but increases computational time.

- The control horizon (M) determines the number of the control actions calculated into the future. Small value of $\mathrm{M}$ makes the controller insensitive of noise. The less $\mathrm{M}$ is useful for controlling the stability of the system while larger $\mathrm{M}$ results in excessive control action and increases the flexibility, but it may lead to instability.

- Prediction and control horizons differ in length. Usually, the prediction horizon is selected to be much longer than the control horizon i.e., $(\mathrm{N} \leq \mathrm{P} \leq \mathrm{M})$.

- Control weighting function $(\lambda)$ is depends on prediction horizon and control horizon. It is set to zero if $\mathrm{P}>>\mathrm{M}$. As the control horizon is increased, the control moves tends to become more aggressive so a larger weight is needed to penalize the control moves.

\section{Results and discussion}

The transfer function model of Wood and Berry distillation column is considered here for the analysis [12].

$$
\begin{aligned}
& {\left[\begin{array}{l}
x_{D} \\
x_{B}
\end{array}\right]=\left[\begin{array}{cc}
\frac{12.8 e^{-s}}{16.7 s+1} & \frac{-18.9 e^{-3 s}}{21 s+1} \\
\frac{6.6 e^{-7 s}}{10.9 s+1} & \frac{-19.4 e^{-3 s}}{14.4 s+1}
\end{array}\right]\left[\begin{array}{l}
L \\
V
\end{array}\right]} \\
& \text { i.e } G(s)=\left[\begin{array}{cc}
\frac{12.8 e^{-s}}{16.7 s+1} & \frac{-18.9 e^{-3 s}}{21 s+1} \\
\frac{6.6 e^{-7 s}}{10.9 s+1} & \frac{-19.4 e^{-3 s}}{14.4 s+1}
\end{array}\right]
\end{aligned}
$$

The plant is having two controlled variables $\mathrm{y}_{1}, \mathrm{y}_{2}$ and two manipulated variables $\mathrm{u}_{1}, \mathrm{u}_{2}$. The two controlled variables are composition of the distillate product $\left(\mathrm{x}_{\mathrm{D}}\right)$ and bottom product $\left(\mathrm{x}_{\mathrm{B}}\right)$. The manipulated variables are reflux (L) and reboiler flow rate $(\mathrm{V})$. The FOPDT model is considered here. The input, reflux flow rate $\mathrm{L}$ and output, distillate product $\mathrm{x}_{\mathrm{D}}$ is considered here to simulate the DMC. Thus, the transfer function model is given by:

$$
\text { i.e } G(s)=\frac{12.8 e^{-s}}{16.7 s+1}
$$

The above transfer function with sampling time 0.5 and after zero order discretization is given by:

$$
G(z)=\frac{0.3776 z^{-3}}{1-0.9705 z^{-1}}
$$

The following are the DMC tuning parameters of the above plant transfer function.

Simulation time $=200 \mathrm{sec}$
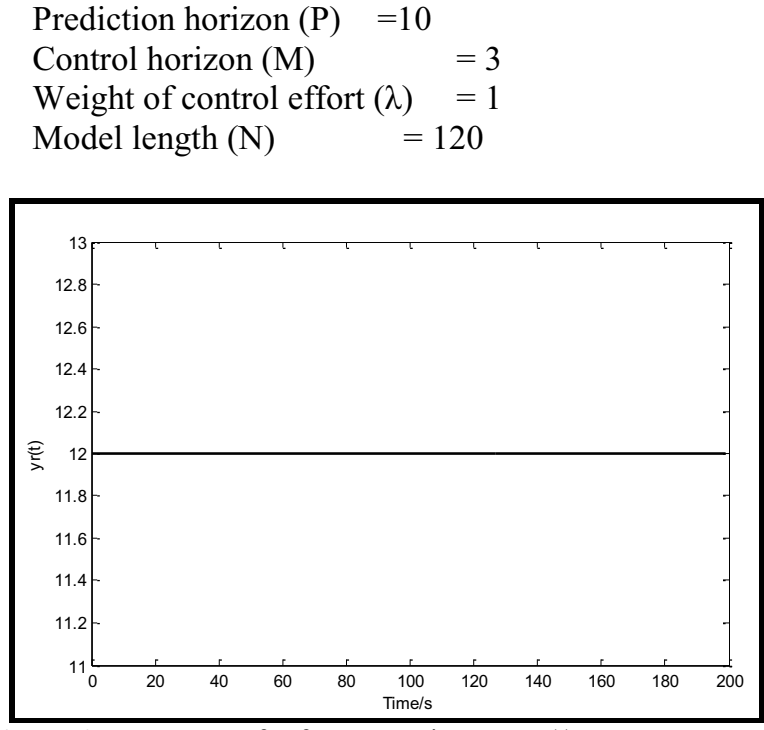

Figure 3. Response of reference trajectory $\mathrm{y}_{\mathrm{r}}(\mathrm{t})$

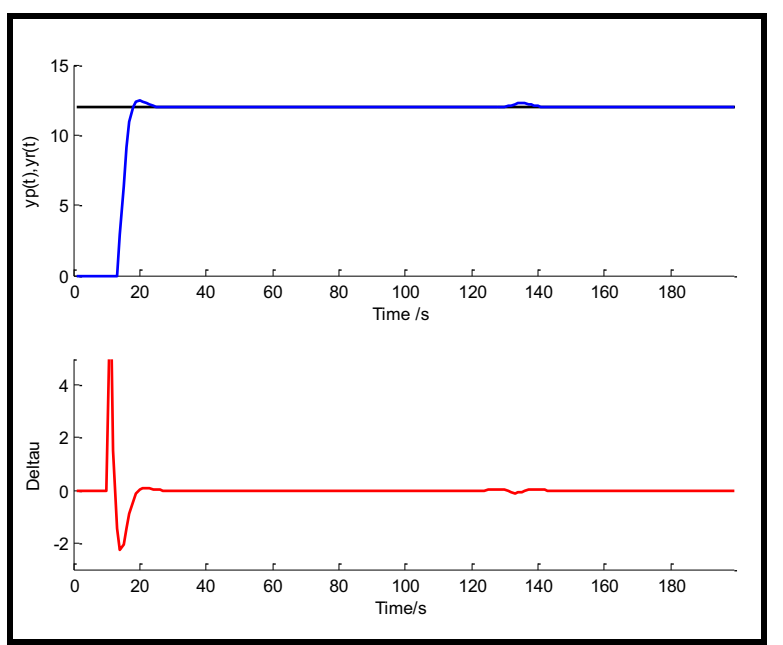

Figure 4. (i) Servo response $\left(\mathrm{y}_{\mathrm{P}}\right)$ and reference trajectory $\left(\mathrm{y}_{\mathrm{r}}\right)$ response (ii) Response of control increment deltau $=\mathrm{K}^{*} \mathrm{e}$

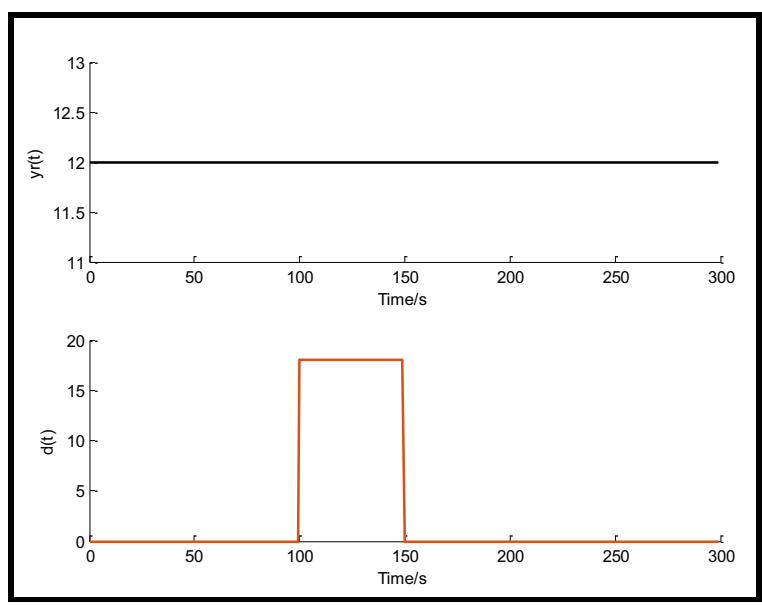

Figure 5. Response of reference trajectory $\mathrm{y}_{\mathrm{r}}(\mathrm{t})$ and disturbance $\mathrm{d}(\mathrm{t})$

Thus, developed an unconstrained DMC algorithm considering one manipulated variable (Reflux flow rate) and controlled variable (Distillate product) of Wood and Berry distillation column. The response reaches the steady state, even in the presence of disturbance. 


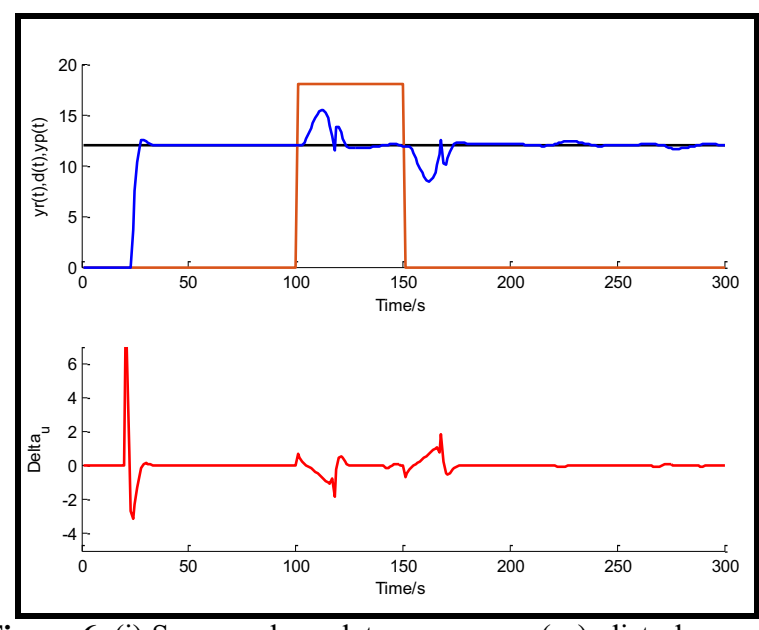

Figure 6. (i) Servo and regulatory response $\left(\mathrm{y}_{\mathrm{P}}\right)$, disturbance $\mathrm{d}(\mathrm{t})$ and reference trajectory $\left(\mathrm{y}_{\mathrm{r}}\right)$ (ii) Response of control increment deltau $=\mathrm{K}^{*} \mathrm{e}$

\section{Conclusions}

The unconstrained DMC implemented for FOPDT model considering one manipulated variable (Reflux flow rate) and a controlled variable (distillate product) of the distillation column. It is a kind of optimization control algorithm, based on prediction model of the step response rolling conduction and marking feedback correction. It is suitable for linear time-invariant system which is asymptotically stable. The tuning parameters ( $\mathrm{P}, \mathrm{M}$ and $\mathrm{N}$ ) place a major role in obtaining better performance. Both the servo and regulatory response is obtained. The simulation result shows good tracking capacity and good disturbance rejection ability. DMC has been found to be satisfactory to control the distillate product purity subject to the specified manipulated variable.

A possible direction for future work is the extension of the method to validate in real time. This topic is currently under investigation and will be reported later. Further, the result of DMC needs to be compared with the DMC-PID in simulation environment

\section{Conflict of interests}

The authors declare that there is no conflict of interests regarding the publication of this paper.

\section{Acknowledgement}

Vinayambika S Bhat to acknowledge the Mangalore Institute of Technology and Engineering, Moodabidri, for sponsoring her Ph.D. programme.

\section{References}

1. Manfred Morari, Jay H. Lee, Carlos E, Garcia, "Model Predictive Control," (2002).

2. William Y. Svreck, Donald P. Mahoney and Brent R. Young, A real time approach to process control, 3 ed., John Wiley and Sons Ltd., (2014).

3. S. Rajasekaran and T. Kannadasan, "A simplified predictive control for a shell and tube heat exchanger," Journal of Engineering Science and Technology, 2, no. 12, (2010).

4. R. D. Kokate and L. M. Waghmare, "An IMC-DMC control for heat exchanger process," International Journal of Computer Applications, 2, no. 26, pp. 96100, (2010).

5. Saniye Ay and Suleyman Karacan, "Decoupling constrained model predictivecontrol of multicomponent packed distillation column," World Applied Sciences Journal, 13, no. 3, pp. 517-530, (2011).

6. R. D. Kokate and L. M. Waghmare, " Review of tuning methods of DMC and performance evaluation with PID algorithms on a FOPDT model," International Journal of Control and Automation, 4, no. 2, pp. 95-110, 2011.

7. S. Srinivasulu Raju, T. S. Darshan and B. Nagendra, "Design of quadratic dynamic matrix control for driven penduum system," International Journal of Electronicsand Communication Engineering, 5, no. 3, pp. 363-370, (2012).

8. Jose Manuel Lopez Guede, Borja Fernandez Gauna, Manuel Grana, Fernado Oterino, "On the influence of the prediction horizon in dynamic matrix control," International Journal of Control Science and Engineering, 3, no. 1, pp. 22-30, 2013.

9. Lin Cong, Xinggao Liu and Chengyu Wang, "Dynamic matrix control of high-purity internal thermally coupled distillation column," Canadian Journal of Chemical Engineering, 92, pp. 696-702, (2014).

10. Manimaran. M, Malaisamy. S, Mohamed rafiq. M, Petchithai. V, Chitra .V. S., Kalanithi. K and Abirami. H, "Parameter identification anddynamic matrix control Design for a nonlinear pilot distillation column," International Journal of ChemTech Research, 7, no. 1, pp. 382-388, (2015).

11. David Di Ruscio, "Model predictive control with integral action: A simple MPC algorithm," Modeling, Identification and Control, 34, no. 3, pp. 119-129, (2013).

12. B. Wanye Bequette, Process Control: Modeling, Design and Simulation, 4 ed., Prentice Hall, (2002). 